Rabaska

Revue d'ethnologie de l'Amérique française

\title{
Claire Quintal (1930-2020). Une amie chère
}

\section{Robert B. Perreault}

Volume 18, 2020

URI : https://id.erudit.org/iderudit/1072924ar

DOI : https://doi.org/10.7202/1072924ar

Aller au sommaire du numéro

Éditeur(s)

Société québécoise d'ethnologie

ISSN

1703-7433 (imprimé)

1916-7350 (numérique)

Découvrir la revue

Citer ce document

Perreault, R. B. (2020). Claire Quintal (1930-2020). Une amie chère. Rabaska, 18, 306-311. https://doi.org/10.7202/1072924ar d'utilisation que vous pouvez consulter en ligne.

https://apropos.erudit.org/fr/usagers/politique-dutilisation/ 


\section{Claire Quintal (1930-2020). Une amie chère}

Le 30 avril 2020, soit deux jours après son $90^{\circ}$ anniversaire de naissance, une championne de la langue et de la culture en Amérique française, Claire Quintal, nous a été enlevée par la Covid-19.

J'ai fait la connaissance de Claire Quintal lors d'un colloque, maintenant légendaire, intitulé « Les Franco-Américains : la promesse du passé et les réalités du présent », organisé par le National Materials Development Center de Bedford au New-Hampshire, en juin 1976. À l'époque, comme bibliothécairearchiviste de l'Association canadoaméricaine de Manchester depuis un

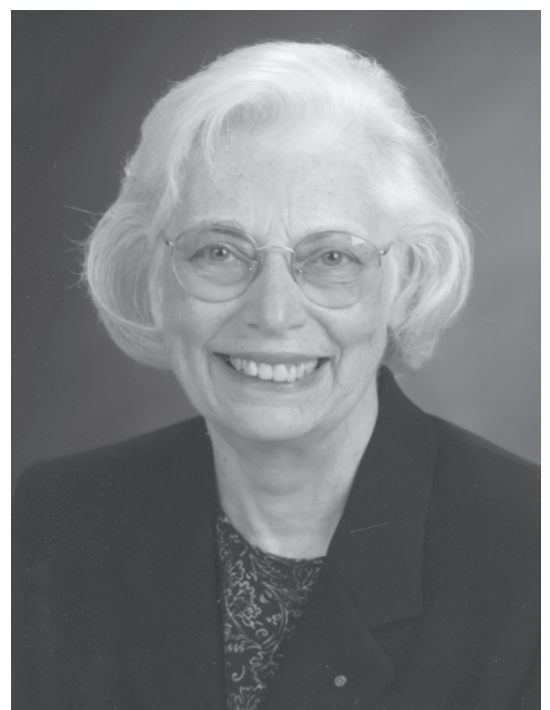

Claire Quintal

Photo : coll. Claire Quintal an et demi, ainsi que représentantobservateur de cette institution au colloque, je faisais mes premiers pas dans le monde du militantisme franco-américain. Jeune - j'avais 25 ans - et naïf quant aux débats dont j'allais être témoin, j'étais choqué par l'explosion de colère que j'appellerais une version franco-américaine de la querelle des «Anciens et des Modernes » en France vers la fin du XVII siècle. C'était donc pour moi mon « baptême du feu».

Malheureusement pour elle, Claire avait accepté, comme elle le disait elle-même, " la tâche de résumer et de réagir aux discours [...] et aux commentaires $[\ldots] »$ du colloque qu'elle trouvait «à la fois mémorables et déplorables ${ }^{1} \gg$. Âgée alors de 46 ans, elle se sentait sans doute coincée entre la

1. Claire Quintal, " Où il s'agit de discourir - Les actes sont à venir » dans Les FrancoAméricains : la promesse du passé et les réalités du présent, Bedford, New-Hampshire, National Materials Development Center for French and Portuguese, 1976, p. 141. 
génération des « anciens », avec leurs idées conservatrices datant de l'époque de la « survivance française », et celle des "modernes », qui favorisaient de nouvelles structures socioculturelles destinées à l'avancement du peuple franco-américain à l'aube du XXI ${ }^{\mathrm{e}}$ siècle. Cependant, de façon soignée, mais aussi franche et directe, Claire a trouvé moyen de remplir sa tâche avec respect et diplomatie, ce qui m'avait beaucoup impressionné.

En juillet de l'année suivante, parmi les activités du festival francoaméricain «C'est si bon » à Lewiston au Maine, on avait organisé un débat littéraire dont le thème était «En quelle langue doit-on écrire ? C Comme auteur d'un premier essai d'importance, One Piece in the Great American Mosaic: The Franco-Americans of New England (1976), on m'a invité à participer au débat avec un petit groupe d'écrivains plus âgés que moi, dont Claire faisait partie. Puisque le débat visait exclusivement l'opposition entre l'écriture en français standard et le parler populaire franco-américain, j'ai proposé - avec un degré d' appréhension, ne voulant pas relancer la querelle de l'année précédente - l'ajout de l'anglais comme langue d'expression littéraire franco-américaine. J'ai donc défendu ma position en rappelant que mon essai en anglais avait eu du succès parmi plusieurs Franco-Américains unilingues anglophones ; certains d'entre eux, enfin capables de comprendre un texte portant sur notre histoire, m'avaient écrit des lettres de remerciement. À mon grand soulagement ainsi qu'à ma joie, Claire m'a appuyé à $100 \%$, disant à l'assistance que j'avais écrit un essai « assez sérieux » qui avait touché une partie de la population qui n'avait pas accès facile à notre littérature puisque celle-ci était écrite, pour la plupart, en français. De nouveau, Claire m'avait beaucoup impressionnée, et de plus, je lui en étais fort reconnaissant.

Au début mars 1978, Claire et moi faisions partie de la délégation de Franco-Américains de la Nouvelle-Angleterre qui assistait au colloque «First Annual Franco-American Conference » à Lafayette en Louisiane. Pendant la réception qui précédait la projection d'un extrait de la pièce théâtrale $L a$ Sagouine de l'auteure acadienne Antonine Maillet, celle-ci, en apercevant Claire dans la foule, l'a saluée et l'a embrassée en l'appelant par son prénom. À son tour, Claire lui a répondu en s'adressant à « Tonine ». Complètement ignorant que Claire et Antonine Maillet se connaissaient aussi intimement, je suis resté étonné devant cette scène. Ensuite, sans hésitation, Claire m'a présenté à $\mathrm{M}^{\mathrm{me}}$ Maillet comme « un de nos jeunes écrivains franco-américains qui promet », et celle-ci a signé mon programme-souvenir de la soirée. Grâce à Claire, ce moment est devenu le clou de mon séjour en Louisiane. Par la suite, lorsque j'ai rencontré $\mathrm{M}^{\mathrm{me}}$ Maillet à deux reprises - l'été suivant à Québec, et douze ans plus tard à Paris - en me voyant, elle m'a reconnu de visage, tout en s'excusant d'avoir oublié mon nom. Cependant, elle se souvenait très bien que Claire m'avait présenté comme Franco-Américain. Encore une 
fois, je me retrouvais redevable à Claire pour sa générosité d'esprit et son encouragement.

Pendant trois semaines vers la fin juin, début juillet 1979, Claire et moi étions parmi une demi-douzaine de Franco-Américains invités par le ministère des Affaires étrangères de France à visiter Paris, la Bretagne et la Bourgogne. Le but de ce séjour était de prendre contact avec quelques fonctionnaires gouvernementaux, mais surtout avec des directeurs de musées, de centres culturels, de maisons d'édition et de divers médias, afin d'établir des rapports et des échanges. Vivant et voyageant ensemble au jour le jour, Claire et moi avons eu maintes discussions à propos de nos vies professionnelles et personnelles, jusqu' au point où, malgré notre différence d'âge, nous sommes devenus bons amis et collègues.

Au cours des années suivantes, Claire m'a invité, à cinq reprises, à donner des conférences sur des thèmes franco-américains divers aux colloques annuels de l'Institut français d'Assumption College à Worcester au Massachusetts, dont elle était la fondatrice et directrice. De plus, en publiant les actes de ces colloques, Claire m'offrait un moyen de faire mieux connaître mes écrits en Amérique française. Nous avons également collaboré au Dictionnaire des auteurs franco-américains de langue française publié en ligne par l'Institut français, pour lequel Claire m'a invité à rédiger les biobibliographies d'une douzaine d'écrivains parmi la centaine qui y paraissent. Avec le passage du temps, j'accumulai une dette énorme de reconnaissance envers elle.

C'est aussi pendant nos années de collaboration que j'ai compris à quel point Claire se dévouait à son travail en faveur des Franco-Américains. Ne dormant que quelques heures, elle passait des nuits blanches à lire, à faire de la recherche, à écrire et à traduire volume après volume du français à l'anglais afin de les rendre accessibles aux Franco-Américains unilingues anglophones, ainsi qu'au public en général.

Le 2 juin 2015, à la demande de la revue Rabaska, je me suis rendu à Worcester où, dans une salle de l'Institut français, j'ai interviewé Claire à propos de sa vie et de sa carrière. Pendant deux séances d'une heure et demie chacune le matin et l'après-midi - avec une pause pour un repas à ses frais dans son restaurant préféré de Worcester - elle fut généreuse de son temps, évoquant, presque sans s'interrompre, des souvenirs qui remontaient jusqu'à son enfance. Pour moi, qui avais fait des entretiens en histoire orale avec des centaines de personnes depuis 1974, c'était un plaisir sans effort, car une seule question pouvait provoquer une réponse d'un quart d'heure ou au-delà ${ }^{2}$.

$* * *$

2. Robert B. Perreault, « Claire Quintal se raconte », Rabaska, vol. 14, 2016, p. 145-169. 
Sur un ton moins officiel et un peu plus personnel, je voudrais maintenant rappeler quelques précieux souvenirs de Claire.

Par contraste avec l'atmosphère formelle qui régnait aux colloques de l'Institut français, on peut juxtaposer l'ambiance détendue des nombreux rassemblements annuels d'artistes et d'écrivains franco-américains. Je n'oublierai jamais, au tout premier " Rassemblement », qui eut lieu dans l'île Mackworth près de Portland au Maine en août 1982, avoir vu Claire y arriver vêtue en jeans ! D'après la condition toute neuve de ce pantalon, il était évident qu'elle se l'était procuré tout spécialement pour l'occasion. Quand je l'ai taquinée à ce propos - « Toi, Claire Quintal, en jeans ? Là, j'aurai tout vu!»-, elle m'a répondu qu'il lui fallait bien s'adapter à la situation. D'après son commentaire, instinctivement, je savais que nous allions avoir une fin de semaine hors pair.

Ayant toujours à la fois plusieurs projets en marche, Claire pouvait facilement passer entre une heure et demie et deux heures au téléphone avec n'importe qui parmi ses nombreux collaborateurs. Parce qu'elle possédait un téléphone fixe ainsi qu'un portable, je ne peux plus compter le nombre de fois, pendant nos conversations téléphoniques, nous avons été interrompus par un appel sur son autre appareil. Cela me faisait toujours rigoler lorsque Claire disait à l'autre personne qu'elle la rappellerait sous peu - ou bien me disait qu'elle devait me quitter pour prendre l'autre l'appel. Dans chaque instance, selon sa décision, je savais laquelle des personnes Claire considérait la plus importante!

À quelques reprises, lors d'un quelconque événement franco-américain qui l'emmenait à Manchester, Claire nous a rendu visite à la maison. Elle a souvent complimenté mon épouse Claudette pour ce qu'elle considérait le bon sens avec lequel elle donnait ses opinions sur la vie en général et la façon dont elle organisait une bonne partie de notre vie quotidienne. Claire reconnaissait bien que j'avais la tête perdue dans mes pensées à propos de mes projets culturels et littéraires - c'est-à-dire, que j'étais souvent « dans la lune ». « Robert t'a bien choisie comme épouse, Claudette », disait-elle avec un petit sourire taquin. «Il reconnaissait qu'il avait besoin d'une femme pratique comme toi pour tâcher de le garder en ligne ».

Si je pouvais faire sourire Claire, je pouvais aussi l'exaspérer, comme la fois qu'elle nous attendait chez elle à une certaine heure et que nous y sommes arrivés au-delà d'une heure en retard. " Claudette, je sais que ce n'est pas ta faute ». Ensuite, elle s'est tournée vers moi. « Et toi, Robert, il faut que tu guettes ton heure ! » m'a-t-elle dit, pointant le doigt comme une mère qui gronde sa petite canaille d'enfant. Mais je pouvais aussi toucher son côté sensible. Ayant lu un de mes contes, «Les mains du père et du fils », Claire m'a avoué qu'à la conclusion, « Robert, tu m'as fait pleurer ». 
Presque annuellement pendant ses années de retraite - c'est-à-dire comme directrice de l'Institut français, mais certainement pas comme auteure et traductrice puisqu'elle a poursuivi ce genre d'activité jusqu'à sa dernière heure - Claire nous invitait à passer une journée entière avec elle à son chalet d'été près de la côte du Maine à York Harbor. Elle nous a révélé que cette maison avait déjà appartenu à une artiste qui s'en servait comme résidence et studio. Fabriquée de bois, ses murs intérieurs ressemblant à ceux d'un camp au fond d'une forêt, elle était à la fois rustique avec un décor artistique, remplie de tableaux et de rayons de livres, en plus d'un balcon qui donnait sur le salon. Cette maison reflétait bien le caractère de sa propriétaire. Soit dehors dans le jardin, soit dans cet environnement intérieur qui inspire la créativité, nous discutions de sujets divers - nos projets littéraires et culturels, nos amis et collègues et leurs propres projets, la vie franco-américaine en général et nos familles respectives. Et ensemble, au cours des années, Claire nous faisait goûter aux fruits de mer et aux poissons dans plusieurs de ses restaurants préférés sur la côte près de chez elle.

Comme institutrice, Claire encourageait les parents et les grands-parents qui prenaient un intérêt tout particulier à l'éducation de leur progéniture, surtout en matières linguistique et culturelle. Pendant notre dernière visite à son chalet d'été, elle nous avait félicités après avoir écouté un enregistrement de notre petite-fille, alors âgée de six ans, qui récitait par cœur « La cigale et la fourmi » de La Fontaine, et notre petit-fils, âgé de quatre ans, nommant, également de mémoire, tous les présidents des États-Unis en ordre chronologique.

Il y a quelques années, un été entier s'étant passé sans que l'invitation annuelle de Claire nous soit lancée, nous avons appris par la suite qu'elle était atteinte d'un cancer. Grave à n'importe quel âge, cette maladie pose davantage de complications et de dangers à un âge avancé. Toutefois, femme forte et courageuse comme nous l'avions toujours connue, Claire a lutté pour vaincre la maladie et s'en sortir vivante.

En 2018, pour des raisons de santé, Claire avait déménagé à la résidence Notre-Dame-du-Lac de Worcester, un ancien couvent converti en appartements pour personnes à la retraite. Nous lui avons rendu visite en novembre 2019. À part plusieurs heures de discussion dans son appartement, toujours entourée de ses livres et de ses objets d'art, elle nous a fait faire le grand tour de la résidence, ceci en dépit du fait qu'elle devait maintenant utiliser une marchette. Elle nous a aussi invités à dîner avec elle dans la salle à manger commune, où la nourriture était excellente et où les autres résidents la saluaient, un par un, comme une bonne amie. 
Certes, Claire était bien logée et elle y semblait heureuse, ce qui nous a bien fait plaisir, à Claudette et moi. En lui disant au revoir, nous envisagions encore plusieurs autres visites chez elle dans son nouvel environnement joyeux. Toutefois, qui aurait deviné, ce jour-là, que ce serait non seulement notre première visite, mais aussi notre dernière ? - car en moins de six mois, Claire n'y serait plus.

Claire me manque énormément, mais au moins, je garde sur les rayons de ma bibliothèque ses écrits et ses traductions, que je peux lire et relire. Et dans mon esprit vivent mes bons souvenirs d'elle. Adieu, chère amie.

Robert B. Perreault Saint Anselm College, Manchester, New-Hampshire 